Empi ri cal determinat i on of equat $i$ on of st at $e$ for zero internal pressure i $n$ rare gas sol i ds and sem-cryst al I i ne pol ymer s

\begin{tabular}{|l|l|}
\hline 著者 & SAEK S, WANG F, TANAKA Y \\
\hline $\begin{array}{l}\text { j our nal or } \\
\text { publ i cat i on t i t l e }\end{array}$ & Pol ymer - Let chwor th \\
\hline vol une & 47 \\
\hline number & 21 \\
\hline page r ange & $7455-7459$ \\
\hline year & $2006-10$ \\
\hline URL & ht t p: //hdl . handl e. net /10098/1161 \\
\hline
\end{tabular}




\title{
Empirical determination of equation of state for zero internal pressure in rare gas solids and semi-crystalline polymers
}

\author{
S. Saeki*, F. Wang, Y. Tanaka \\ Department of Materials Science and Engineering, Fukui University, Fukui 910-8507, Japan
}

\begin{abstract}
An equation of state for zero internal pressure in rare gas solids and semi-crystalline polymers has been determined based on the empirical functions of thermal pressure coefficient $\gamma_{V}$ with respect to volume at constant pressure. The experimental data of $P V T$ over wide range of temperature and pressure published by Anderson and Swenson and Syassen and Holzapfel for rare gas solids and Olabisi and Simha and Zoller for semi-crystalline polymers are used to evaluate $\gamma_{V}$. The function of $\gamma_{V}$ with respect to volume determined at constant pressure is given by $\gamma_{V}=$ $A\left\{\left(V-V_{0}\right) / V\right\}^{\varepsilon}\left(V_{0} / V\right)^{1+c} \exp \left\{-c\left(V-V_{0}\right) / V\right\}$ where $V_{0}$ is the volume at $0 \mathrm{~K}, A, \varepsilon$ and $c$ are constants. The function of internal pressure $P_{\mathrm{i}}=\gamma_{V} T-P$ with respect to temperature at constant pressure is determined by converting the function of $\gamma_{V}(V)$ to a function of temperature $\gamma_{V}(T)$. An empirical equation of state for zero internal pressure determined by pressure $P^{*}$, volume $V^{*}$ and temperature $T^{*}$ at which $P_{\mathrm{i}}=0$ is expressed by $P^{*} V^{*} / \mathrm{R} T^{*}=C^{*}-D^{*} V^{*}$ for rare gas and semi-crystalline polymer where $C^{*}$ and $D^{*}$ are constants. The practical meaning of the equation of state for $P_{\mathrm{i}}=0$ in the semi-crystalline polymers has been discussed.

(C) 2006 Elsevier Ltd. All rights reserved.
\end{abstract}

Keywords: Internal pressure; Thermal pressure coefficient; Semi-crystalline polymer

\section{Introduction}

The main purposes of the experimental and theoretical studies on the equation of state have been the accurate determination of $P V T$ over wide range of pressure and temperature and their theoretical interpretation. The theoretical equation of states for semi-crystalline polymers have been derived by Pastine [1,2], Simha and Jain [3], Midha and Nanda [4], which give theoretical prediction of $P V T$ data quantitatively. The extensive experimental studies of the $P V T$ for the semicrystalline polymers have been done by Olabisi and Simha [5] and Zoller [6] over the wide range of temperature and pressure. On the other hand the experimental and theoretical studies of PVT for rare gas solids have been done by Anderson and Swenson [7], Syassen and Holzapfel [8] and Bernardes

\footnotetext{
* Corresponding author. Tel: +81776 27 8622; fax: +81 776278767 .

E-mail address: saeki@matse.fukui-u.ac.jp (S. Saeki).
}

and Swenson [9]. Based on these experimental PVT data it becomes possible to determine a function of isothermal compressibility and thermal expansion coefficient with respect to pressure and temperature, respectively. In previous works [10-15] we have derived a simple equation that enables an accurate determination of isothermal compressibility and thermal expansion coefficient. In this work we have determined an empirical function of thermal pressure coefficient with respect to volume at constant pressure over wide range of pressure from which the function of internal pressure $P_{\mathrm{i}}=\gamma_{V} T-P$ with respect to temperature can be determined. The experimental data of $P_{\mathrm{i}}$ against temperature at various constant pressures provide enough way to determine a characteristic pressure $P^{*}$, volume $V^{*}$ and temperature $T^{*}$ at which the $P_{\mathrm{i}}$ becomes zero. The main purpose of this work is to determine the equation of state for zero internal pressure in rare gas solids and semi-crystalline polymers by the experimental data of $P^{*}, V^{*}$ and $T^{*}$. 


\section{Empirical determination of thermal pressure coefficient and intermal pressure in rare gas and semi-crystalline polymers from $P V T$ data}

Two steps are involved in the determination of thermal pressure coefficient. First, the isothermal compressibility is determined from the $P-V$ data at constant temperature. Second, the thermal expansion coefficient is evaluated from $V-T$ data at constant pressure. The two empirical equations derived in the previous work $[12,15]$ are used in this work. The first equation is for the function of volume $V(P, T)$ with respect to pressure at constant temperature and is given by

$$
\begin{aligned}
& V\left(P_{0}, T\right) / V(P, T) \\
& \quad=\left[\left\{P+P_{x}(T)\right\} /\left\{P_{0}+P_{x}(T)\right\}\right]^{m_{x}} \quad T=\mathrm{constant}
\end{aligned}
$$

where the volume $V\left(P_{0}, T\right)$ is $V$ at $\left(P_{0}=0\right.$ bar,T $), P_{x}(T)$ is a function of temperature and $m_{x}$ is a constant. The second equation is a function of temperature with respect to volume $T(V)$ at constant pressure and is given by

$\ln \left(T / T_{1}\right)=B\left\{\left(V-V_{1}\right) / V\right\}^{n_{x}} \quad P=\mathrm{constant}$

where $T_{1}=1 \mathrm{~K}, V_{1}$ is the volume at $T_{1}=1 \mathrm{~K}$. The values of $B$ and $n_{x}$ are constant. The compressibility $\beta_{T}$ is calculated from Eq. (1) as

$\beta_{T}=m_{x} /\left\{P+P_{x}(T)\right\}$

The thermal expansion coefficient $\alpha_{p}$ obtained from Eq. (2) is expressed by

$\alpha_{P}=\left(V-V_{1}\right) /\left(n_{x} V_{1} T \ln T\right)$

From the thermal pressure coefficient $\gamma_{V}$ defined by $\gamma_{V}=$ $\alpha_{P} / \beta_{T}$ the internal pressure $P_{\mathrm{i}}$ is obtained as $P_{\mathrm{i}}=\gamma_{V} T-P$. The typical comparisons between the experimental data and values calculated by Eqs. (1) and (2) are shown in Table 1 where the difference between the specific volumes in linear polyethylene [5] for the $P-V$ isotherm is $0.007 \%$ in average over the pressure range up to 2000 bar, while the difference for the $T-V$ isobar is $0.064 \%$ in average over the temperature range of $19.5-129^{\circ} \mathrm{C}$ in semi-crystalline state of polyethylene.

We have selected the equations for the determination of $\beta_{T}$ and $\alpha_{P}$ towards an accurate determination of $\gamma_{V}$. For example, Eq. (1) is used for the determination of $\beta_{T}$, while Eq. (2) is used for $\alpha_{P}$. The $\alpha_{P}$ may be determined by Eq. (1) with less accuracy because $m_{x}$ is not constant. The values of $m_{x}$ and $P_{x}(T)$ used to determine $\beta_{T}$ for rare gas solids are listed in Table 2, while those for semi-crystalline polymers are taken from literature [13].

It is somewhat unfortunate that Eqs. (3) and (4) for $\alpha_{P}$ and $\beta_{T}$ cannot give an accurate function for $\gamma_{V}=\alpha_{P} / \beta_{T}$ because $m_{x}$ varies on temperature slightly and irregularly and the function $P_{x}(T)$ with respect to temperatures is not smooth enough to obtain a simple function of temperature. We have tried to determine a simple empirical function of $\gamma_{V}$ with respect to volume at constant pressure. The mathematical procedure for the determination of $\gamma_{V}=(\partial S / \partial V)_{T}$ from entropy $S$ has been described elsewhere $[14,15]$. One of the useful functions of $\gamma_{V}$ found by the mathematical procedure is given by

$\gamma_{V}=A\left\{\left(V-V_{0}\right) / V\right\}^{\varepsilon}\left(V_{0} / V\right)^{1+c} \exp \left\{-c\left(V-V_{0}\right) / V\right\}$

where $V_{0}$ is a volume at $T=0 \mathrm{~K}$. We assumed in the calculation that $V_{0}$ is equal to $V_{1}$ at $T_{1}=1 \mathrm{~K}$ in Eq. (4). In the mathematical procedure $[14,15]$ we used an assumption that $\gamma_{V}(V)$ is a function of the variable $\left(V-V_{0}\right) / V$ and is expressed by its power law, which enables a transformation of $\gamma_{V}(V)$ to $\gamma_{V}(T)$ easily as is described below. The variable $\left(V-V_{0}\right) / V$ is a measure of free volume fraction in the solid state and plays an important role in the function of thermal pressure coefficient with respect to volume. It is found that Eq. (5) allows good prediction of $\gamma_{V}(V)$ at constant pressure. The constants $A, \varepsilon$ and $c$ in Eq. (5) determined by fitting the experimental data of $\gamma_{V}$ are listed in Table 3 for rare gas solids and Table 4 for semi-crystalline polymers where values of volume $V_{0}$ at $0 \mathrm{~K}$ for various pressure are also listed. Typical data of $\gamma_{V}(\mathrm{~V})$ for polypropylene are shown in Fig. 1 where the line is calculated by Eq. (5).

Table 1

Comparison between the experimental data $\left(V_{\text {exp }}\right)$ of $P V T$ for polyethylene [5] and values $\left(V_{\text {cal }}\right)$ calculated by Eq. (1) with $m_{x}=0.0909, P_{x}=3470$ bar,

\begin{tabular}{|c|c|c|c|c|c|c|c|}
\hline \multicolumn{4}{|c|}{ Volume $(\mathrm{cc} / \mathrm{g})$ at various pressure at $55.8^{\circ} \mathrm{C}$} & \multicolumn{4}{|c|}{ Volume $(\mathrm{cc} / \mathrm{g})$ at various temperature at 1200 bar } \\
\hline$P$ (bar) & $V_{\exp }(\mathrm{cc} / \mathrm{g})$ & $V_{\text {tal }}(\mathrm{cc} / \mathrm{g})$ & $V_{\text {exp }}-V_{\text {cal }}$ & Temp ( $\left.{ }^{\circ} \mathrm{C}\right)$ & $V_{\exp }(\mathrm{cc} / \mathrm{g})$ & $V_{\text {cal }}(\mathrm{cc} / \mathrm{g})$ & $V_{\text {exp }}-V_{\text {cal }}$ \\
\hline 1 & 1.0341 & 1.0341 & - & 19.5 & 0.9981 & 0.9981 & - \\
\hline 100 & 1.0315 & 1.0315 & - & 32.3 & 1.0017 & 1.0004 & 0.0013 \\
\hline 200 & 1.0289 & 1.0289 & - & 47.6 & 1.0048 & 1.0037 & 0.0011 \\
\hline 300 & 1.0264 & 1.0264 & - & 55.8 & 1.0064 & 1.0057 & 0.0007 \\
\hline 400 & 1.0240 & 1.0239 & 0.0010 & 66.1 & 1.0091 & 1.0085 & 0.0006 \\
\hline 600 & 1.0192 & 1.0192 & - & 76.4 & 1.0117 & 1.0116 & 0.0001 \\
\hline 800 & 1.0148 & 1.0148 & - & 84.8 & 1.0139 & 1.0144 & -0.0005 \\
\hline 1000 & 1.0105 & 1.0106 & -0.0001 & 94.5 & 1.0174 & 1.0180 & -0.0006 \\
\hline 1200 & 1.0064 & 1.0066 & -0.0002 & 104.5 & 1.0221 & 1.0220 & 0.0001 \\
\hline 1400 & 1.0028 & 1.0028 & - & 115.5 & 1.0290 & 1.0269 & 0.0021 \\
\hline 1600 & 0.9993 & 0.9991 & 0.0002 & 128.9 & 1.0337 & 1.0336 & 0.0001 \\
\hline 1800 & 0.9957 & 0.9956 & 0.0001 & & & & \\
\hline 2000 & 0.9922 & 0.9922 & - & & & & \\
\hline
\end{tabular}
$V\left(P_{0}=1 \mathrm{bar}, T=329 \mathrm{~K}\right)=1.0341(\mathrm{cc} / \mathrm{g})$ and Eq. $(2)$ with $B=6.741, V(P=1200 \mathrm{bar}, T=1 \mathrm{~K})=0.9876(\mathrm{cc} / \mathrm{g}), n_{x}=0.0376$ 
Table 2

The constants in Eq. (1) for rare gas solids determined at constant temperatures

\begin{tabular}{|c|c|c|c|c|}
\hline & $T(\mathbf{K})$ & $V_{0, T}(\mathrm{cc} / \mathrm{g})$ & $m_{x}$ & $P_{x}(T)$ (bar) \\
\hline \multirow[t]{6}{*}{$\operatorname{Ar}[7]$} & 0 & 0.5646 & 0.1513 & 4459 \\
\hline & 4 & 0.5646 & 0.1559 & 4643 \\
\hline & 20 & 0.5668 & 0.1548 & 4442 \\
\hline & 40 & 0.5766 & 0.1520 & 3749 \\
\hline & 60 & 0.5914 & 0.1456 & 2858 \\
\hline & 77 & 0.6079 & 0.1421 & 2222 \\
\hline \multirow[t]{9}{*}{$\operatorname{Kr}[7]$} & 0 & 0.3233 & 0.1474 & 5001 \\
\hline & 4 & 0.3234 & 0.1510 & 5178 \\
\hline & 20 & 0.3249 & 0.1463 & 4698 \\
\hline & 40 & 0.3294 & 0.1450 & 4142 \\
\hline & 60 & 0.3350 & 0.1454 & 3700 \\
\hline & 77 & 0.3405 & 0.1414 & 3151 \\
\hline & 90 & 0.3456 & 0.1395 & 2740 \\
\hline & 100 & 0.3502 & 0.1392 & 2480 \\
\hline & 110 & 0.3550 & 0.1394 & 2261 \\
\hline \multirow[t]{11}{*}{$\mathrm{Xe}[7]$} & 0 & 0.2645 & 0.1420 & 5191 \\
\hline & 4 & 0.2646 & 0.1464 & 5387 \\
\hline & 20 & 0.2653 & 0.1470 & 5298 \\
\hline & 40 & 0.2676 & 0.1454 & 4881 \\
\hline & 60 & 0.2708 & 0.1451 & 4464 \\
\hline & 77 & 0.2740 & 0.1405 & 3900 \\
\hline & 80 & 0.2743 & 0.1411 & 3897 \\
\hline & 100 & 0.2782 & 0.1417 & 3556 \\
\hline & 120 & 0.2819 & 0.1426 & 3290 \\
\hline & 140 & 0.2868 & 0.1390 & 2751 \\
\hline & 159 & 0.2932 & 0.1311 & 2062 \\
\hline
\end{tabular}

As far as we know there is no data available for low temperature range near $0 \mathrm{~K}$ for polypropylene, as is shown in Fig. 1. However, in the determination of the empirical function of $\gamma_{V}(V)$ we have used hypothetical point obtained from a thermodynamic condition that $\gamma_{V}\left(V_{0}\right)=0$ at $V_{0}$.

\section{Results}

The determination of empirical function of $\gamma_{V}(T)$ has been carried out by using Eq. (5). The function of $\gamma_{V}(V)$ in Eq. (5) is converted into $\gamma_{V}(T)$ by using a relation $\ln \left(T / T_{1}\right)=$ $B\left\{\left(V-V_{0}\right) / V\right\}^{n_{x}}$ in Eq. (2). The empirical function of $\gamma_{V}(T)$ is expressed by

$$
\begin{aligned}
\gamma_{V}= & A\left(\frac{\ln \left(\frac{T}{T_{1}}\right)}{B}\right)^{\varepsilon / n_{x}}\left[1-\left(\frac{\ln \left(\frac{T}{T_{1}}\right)}{B}\right)^{1 / n_{x}}\right]^{1+c} \\
& \times \exp \left[-c\left(\frac{\ln \left(\frac{T}{T_{1}}\right)}{B}\right)^{1 / n_{x}}\right]
\end{aligned}
$$

Eq. (6) allows the determination of $P_{\mathrm{i}}=\gamma_{V} T-P$ as a function of temperature at constant pressure. Therefore a temperature $T^{*}$ at which $P_{\mathrm{i}}=0$ can be determined at $P=P^{*}$. The typical experimental values of $P_{\mathrm{i}}=\gamma_{V} T-P$ for solid xenon are plotted against temperature at constant pressure in Fig. 2 where a good agreement between the experimental data and the lines calculated by $\mathrm{Eq}$. (6) are obtained over wide range of temperatures. The value of $V^{*}$ for $P_{\mathrm{i}}=0$ is also determined by $T^{*}, P^{*}$ and Eq. (2). All constants in Eq. (6) and values of $P^{*}, V^{*}$ and $T^{*}$ evaluated are listed in Tables 3 and 4 .

We have found that a relation between $P^{*}, V^{*}$ and $T^{*}$ is expressed by an empirical equation $V^{*-1}=a_{0}+b_{0}\left(P^{*} / T^{*}\right)$. From this equation we have derived an equation that

$P^{*} V^{*} / \mathrm{R} T^{*}=C^{*}-D^{*} V^{*}$

This is an empirical equation for PVT surface with zero internal pressure. Typical data of $P^{*}, V^{*}, T^{*}$ for polyethylene in three dimensions are shown in Fig. 3. The constants $C^{*}$ and $D^{*}$ are listed in Table 5. The thermal pressure coefficient for the equation of state with $P_{\mathrm{i}}=0$ is given using Eq. (7) by

$\gamma_{V}^{*}=\frac{\mathrm{R}\left(C^{*}-D^{*} V^{*}\right)}{V^{*}}$

Table 3

\begin{tabular}{|c|c|c|c|c|c|c|c|c|c|c|c|}
\hline Rare gas solid & $P$ (bar) & $A(\mathrm{bar} / \mathrm{K})$ & $c$ & $\varepsilon$ & $B$ & $n_{x}$ & $V_{1}=V_{0}(\mathrm{cc} / \mathrm{g})$ & $P *($ bar $)$ & $V^{*}(\mathrm{cc} / \mathrm{mol})$ & $T^{*}(\mathrm{~K})$ & $P^{*} V * / \mathrm{R} T^{*}$ \\
\hline \multirow[t]{4}{*}{$\operatorname{Ar}[7]$} & 1 & 118.93 & 3.593 & 0.3784 & 6.169 & 0.1327 & 0.5646 & & & & \\
\hline & 500 & 130.63 & 4.455 & 0.3697 & 6.433 & 0.1376 & 0.5554 & 500 & 22.35 & 25.73 & 5.22 \\
\hline & 1000 & 116.15 & 4.596 & 0.3480 & 6.647 & 0.1404 & 0.5474 & 1000 & 22.44 & 42.36 & 6.31 \\
\hline & 1500 & 107.83 & 4.699 & 0.3353 & 6.823 & 0.1421 & 0.5402 & 1500 & 22.22 & 61.80 & 6.48 \\
\hline \multirow[t]{5}{*}{$\mathrm{Kr}[7]$} & 1 & 155.65 & 5.008 & 0.4240 & 6.711 & 0.1473 & 0.3233 & & & & \\
\hline & 500 & 124.59 & 5.008 & 0.3703 & 6.943 & 0.1512 & 0.3188 & 500 & 26.91 & 26.99 & 5.99 \\
\hline & 1000 & 110.04 & 5.077 & 0.3418 & 7.136 & 0.1535 & 0.3147 & 1000 & 26.81 & 44.52 & 7.22 \\
\hline & 1500 & 97.23 & 5.021 & 0.3140 & 7.299 & 0.1550 & 0.3110 & 1500 & 26.79 & 64.76 & 7.46 \\
\hline & 2000 & 88.37 & 4.960 & 0.2932 & 7.446 & 0.1560 & 0.3077 & 2000 & 26.85 & 90.44 & 7.14 \\
\hline \multirow[t]{8}{*}{$\mathrm{Xe}[7]$} & 1 & 86.76 & 4.020 & 0.3400 & 7.328 & 0.1542 & 0.2645 & & & & \\
\hline & 500 & 65.13 & 4.518 & 0.2706 & 7.537 & 0.1570 & 0.2611 & 500 & 34.52 & 31.60 & 6.57 \\
\hline & 1000 & 63.89 & 4.429 & 0.2673 & 7.614 & 0.1550 & 0.2580 & 1000 & 34.43 & 55.36 & 7.48 \\
\hline & 1500 & 58.72 & 4.883 & 0.2465 & 7.892 & 0.1608 & 0.2552 & 1500 & 34.45 & 83.46 & 7.45 \\
\hline & 2000 & 56.15 & 4.849 & 0.2369 & 8.008 & 0.1610 & 0.2526 & 2000 & 34.55 & 117.63 & 7.07 \\
\hline & 2500 & 57.41 & 5.289 & 0.2381 & 8.164 & 0.1621 & 0.2501 & 2500 & 34.87 & 169.95 & 6.17 \\
\hline & 3000 & 56.82 & 5.479 & 0.2348 & 8.285 & 0.1636 & 0.2479 & 3000 & & & \\
\hline & 4000 & 56.72 & 5.857 & 0.2366 & 8.526 & 0.1652 & 0.2440 & & & & \\
\hline
\end{tabular}

The constants in Eqs. (2) and (5) for rare gas solids determined at constant pressure and the values of $P^{*}, V^{*}$ and $T^{*}$ and its ratio $P^{*} V^{*} / \mathrm{R} T^{*}$ in Eq. (7) 
Table 4

The constants in Eqs. (2) and (5) for semi-crystalline polymer determined at constant pressure and the values of $P^{*}, V^{*}$ and $T^{*}$ and its ratio $P^{*} V^{*} / \mathrm{R} T^{*}$ in Eq. (7)

\begin{tabular}{|c|c|c|c|c|c|c|c|c|c|c|c|c|}
\hline Polymer solid & $M_{0}(\mathrm{~g} / \mathrm{mol})$ & $P($ bar $)$ & $A(\mathrm{bar} / \mathrm{K})$ & $c$ & $\varepsilon$ & $B$ & $n_{x}$ & $V_{1}=V_{0}(\mathrm{cc} / \mathrm{g})$ & $P *(b a r)$ & $V^{*}(\mathrm{cc} / \mathrm{g})$ & $T^{*}(\mathrm{~K})$ & $P^{*} V * / \mathrm{R} T^{*}$ \\
\hline \multirow[t]{5}{*}{ PP [18] } & 42.08 & 1 & 224.3 & 7.209 & 0.7618 & 6.932 & 0.0405 & 1.0793 & & & & \\
\hline & & 500 & 241.9 & 8.216 & 0.7586 & 7.027 & 0.0580 & 1.0591 & 500 & 1.0629 & 158.6 & 1.70 \\
\hline & & 1000 & 90.7 & 5.443 & 0.5238 & 7.095 & 0.0561 & 1.0530 & 1000 & 1.0580 & 191.8 & 2.79 \\
\hline & & 1500 & 178.9 & 7.313 & 0.6706 & 7.079 & 0.0508 & 1.0484 & 1500 & 1.0533 & 219.3 & 3.65 \\
\hline & & 2000 & 159.1 & 5.304 & 0.6329 & 6.932 & 0.0405 & 1.0456 & 2000 & 1.0490 & 244.1 & 4.35 \\
\hline \multirow[t]{4}{*}{ PE [5] } & 28.05 & 1 & 87.6 & 3.187 & 0.4207 & 6.637 & 0.0383 & 1.0036 & & & & \\
\hline & & 400 & 472.8 & 11.275 & 0.7706 & 6.715 & 0.0404 & 0.9965 & 400 & 0.9976 & 163.7 & 0.82 \\
\hline & & 800 & 283.0 & 8.188 & 0.6928 & 6.841 & 0.0453 & 0.9883 & 800 & 0.9908 & 184.9 & 1.45 \\
\hline & & 1200 & 101.5 & 2.597 & 0.4816 & 6.741 & 0.0376 & 0.9876 & 1200 & 0.9900 & 216.7 & 1.85 \\
\hline \multirow[t]{4}{*}{ HMWPE [5] } & 28.05 & 1 & 251.0 & 4.296 & 0.7411 & 6.512 & 0.0379 & 1.0495 & 0 & & & \\
\hline & & 400 & 493.9 & 5.826 & 0.8880 & 6.553 & 0.0384 & 1.0398 & 400 & 1.0409 & 149.0 & 0.94 \\
\hline & & 800 & 255.9 & 3.677 & 0.7308 & 6.622 & 0.0409 & 1.0291 & 800 & 1.0329 & 193.6 & 1.44 \\
\hline & & 1200 & 141.9 & 1.908 & 0.5822 & 6.694 & 0.0436 & 1.0197 & 1200 & 1.0244 & 198.9 & 2.09 \\
\hline \multirow[t]{4}{*}{ P4MPI $[6]^{3 \mathrm{t}}$} & 84.15 & 1 & 73.1 & 4.377 & 0.5851 & 7.068 & 0.0674 & 1.1570 & 0 & & & \\
\hline & & 200 & 42.1 & 3.951 & 0.4011 & 7.346 & 0.0841 & 1.1372 & 200 & 1.1388 & 68.0 & 3.32 \\
\hline & & 400 & 336.6 & 7.824 & 0.9347 & 7.698 & 0.1076 & 1.1130 & 400 & 1.1238 & 106.8 & 4.18 \\
\hline & & 600 & 123.2 & 6.369 & 0.6448 & 7.811 & 0.1105 & 1.1086 & 600 & 1.1197 & 109.0 & 6.12 \\
\hline \multirow[t]{4}{*}{ NY66 [18] } & 226.31 & 1 & & 5.359 & 1.0758 & 6.968 & 0.0546 & 0.8542 & & & & \\
\hline & & 500 & 133.6 & 2.824 & 0.6556 & 7.025 & 0.0553 & 0.8448 & 500 & 0.8475 & 165.9 & 6.95 \\
\hline & & 1000 & 155.6 & 2.742 & 0.6661 & 7.019 & 0.0521 & 0.8398 & 1000 & 0.8445 & 211.8 & 10.85 \\
\hline & & 1500 & 71.3 & 0.747 & 0.4360 & 7.021 & 0.0502 & 0.8340 & 1500 & 0.8381 & 216.2 & 15.83 \\
\hline \multirow[t]{6}{*}{ POM [19 ${ }^{a}$} & 30.03 & 1 & 541.9 & 7.702 & 0.8190 & 6.801 & 0.0454 & 0.6929 & & & & \\
\hline & & 400 & 315.1 & 6.206 & 0.6844 & 6.921 & 0.0508 & 0.6874 & 400 & 0.6882 & 134.5 & 0.73 \\
\hline & & 800 & $1.446 \times 10^{3}$ & 10.373 & 1.0335 & 6.965 & 0.0515 & 0.6841 & 800 & 0.6868 & 187.3 & 1.04 \\
\hline & & 1200 & $14.31 \times 10^{3}$ & 15.559 & 1.6319 & 7.261 & 0.0680 & 0.6742 & 1200 & 0.6812 & 204.4 & 1.41 \\
\hline & & 1600 & $10.71 \times 10^{3}$ & 16.437 & 1.4898 & 7.078 & 0.0540 & 0.6789 & 1600 & 0.6848 & 238.6 & 1.63 \\
\hline & & 2000 & $190.1 \times 10^{3}$ & 24.444 & 2.1673 & 7.115 & 0.0549 & 0.6763 & 2000 & 0.6848 & 267.8 & 1.81 \\
\hline
\end{tabular}

$\mathrm{M}_{0}: M_{\mathrm{w}}$ of monomer unit, PP: polypropylene, PE: polyethylene, HMWPE: high molecular weight polyethylene, P4MPI: poly-4-methyl-pentene-1, NY66: Nylon66, POM: polyoxymethylene.

a The units of pressure for $P, A$ and $P^{*}$ are $\mathrm{kg} / \mathrm{cm}^{2}$.

where $\gamma_{V}^{*}$ is defined by $\left(\partial P^{*} / \partial T^{*}\right)_{V^{*}}$ It is obvious from Eqs. (7) and (8) that the internal pressure $P_{\mathrm{i}}^{*}$ is zero at every points on the $P^{*} V^{*} T^{*}$ surface.

\section{Discussions}

According to the third law of thermodynamics the entropy of the material approaches to a constant value $S_{0}$ in the limit of

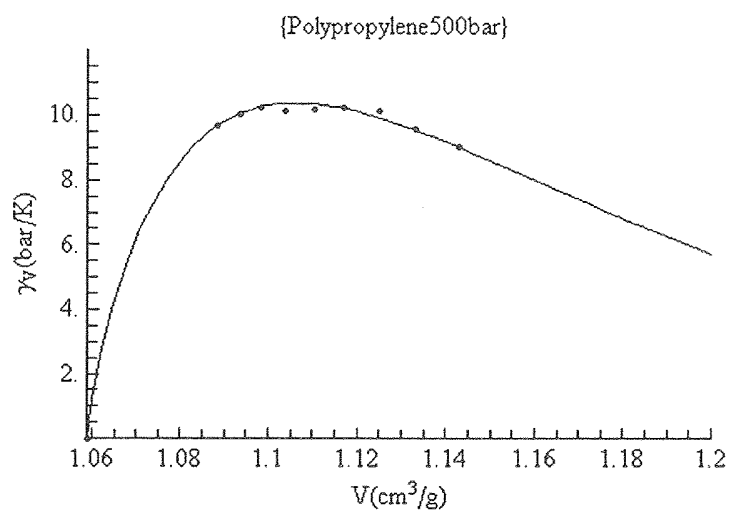

Fig. 1. $\gamma_{V}$ vs. $V$ plot for polypropylene at 500 bar. The experimental data are taken from Ref. [18].
$0 \mathrm{~K}$, which does not depend on volume and pressure. Therefore $(\partial S / \partial P)_{T} \rightarrow 0$ at $T \rightarrow 0$, which gives $(\partial V / \partial T)_{P}=V \alpha_{P}$ $=0$ due to the Maxwell relation and then $\alpha_{P} \rightarrow 0$ and $\gamma_{V} \rightarrow 0$ at $T \rightarrow 0$ [20]. This is a criterion in deriving the empirical function of $\gamma_{V}$ in Eq. (5) where $\gamma_{V}$ is zero at $V \rightarrow V_{0}$ through $T \rightarrow 0$. An existence of empirical equation of state for $P^{*} V^{*} T^{*}$ with $P_{\mathrm{i}}=0$ is confirmed by the two thermodynamic facts that $P_{\mathrm{i}}=\gamma_{V} T-P<0$ at $T \rightarrow 0$ under high

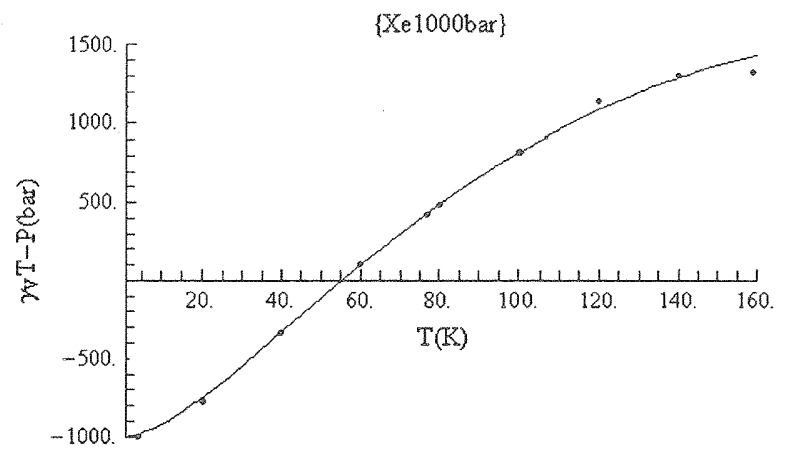

Fig. 2. $\gamma_{V} T-P$ vs. $T$ plot for Xenon at 1000 bar. The experimental data are taken from Ref. [7]. 


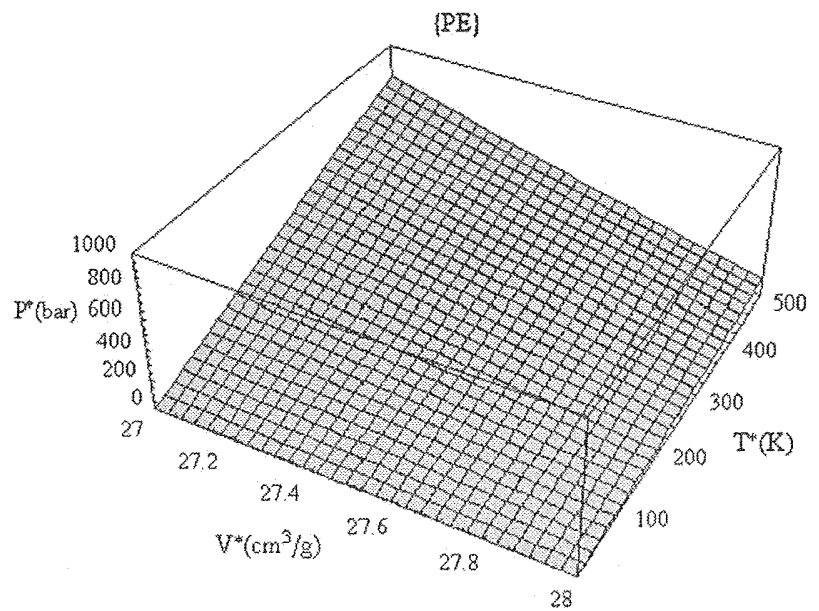

Fig. 3. $P^{*} V^{*} T^{*}$ surface for the equation of state with $P_{\mathrm{i}}=0$ in polyethylene calculated by Eq. (7) and values of $C^{*}$ and $D^{*}$ in Table 5 .

pressure $P>1$ since $\gamma_{V}$ is zero at $T \rightarrow 0$ and the experimental result that $P_{\mathrm{i}}>0$ at higher temperature such as $T_{1}>0$. Therefore $P_{i}^{*}=\gamma_{V}^{*} T^{*}-P^{*}=0$ exists between $0<T^{*}<T_{1}$ (see Fig. 2).

It is interesting to discuss the practical meaning of the equation of state for zero internal pressure for solids. The three dimension $P^{*} V^{*} T^{*}$ plot is shown in Fig. 3 where the region above the $P^{*} V^{*} T^{*}$ surface correspond to high pressure region where $\gamma^{*} T^{*}=P^{*}<P$ or $P_{\mathrm{i}}<0$, while the region below the surface shows $P_{\mathrm{i}}>0$ and the surface shows $\gamma^{*} T^{*}=P^{*}$ or $P_{\mathrm{i}}=0$. The internal pressure $P_{\mathrm{i}}$ is related to the internal energy $U$ by

$P_{\mathrm{i}}=(\partial U / \partial V)_{T}$

No internal energy change occurs by changing the volume at constant temperature on the $P^{*} V^{*} T^{*}$ surface due to the cancellation between the external pressure $P$ and thermal pressure $\gamma_{V} T$. The surface of $P^{*} V^{*} T^{*}$ with $(\partial U / \partial V)_{T}=0$ may be meaningful in a processing such that a rare component is introduced into the inside of solid material without energy change.

Pastine $[1,2]$ has derived the equation of state for polymer solid where a pressure consists of two factors that $0 \mathrm{~K}$ isotherm and thermal pressure contribution above $0 \mathrm{~K}$ and derived the equation of state for polyethylene of arbitrary crystallinity. In this work we have not discussed the effect of crystallinity on the equation of state. The thermal pressure coefficient evaluated in this work and constants obtained are limited for the semi-crystalline polymer from which the data were obtained.

It is interesting to discuss about the theoretical works of $\gamma_{V}$ with respect to volume or temperature at constant pressure. The equation of state for polymer solids derived by Hartmann and Haque [16] is given in reduced form by $\bar{P} \bar{V}=\bar{T}^{3 / 2}-\ln \bar{V}$ which can predict a maximum of $\gamma_{V}$ with respect to volume
Table 5

The constant in the Eq. (7) for rare gas solids and semi-crystalline polymers

\begin{tabular}{lcc}
\hline Solid & $C^{*}$ & $D^{*}(\mathrm{~mol} / \mathrm{cc})$ \\
\hline Xe & 109.3 & 2.960 \\
$\mathrm{Kr}$ & 308.4 & 11.230 \\
$\mathrm{Ar}$ & 240.6 & 10.540 \\
POM & 188.6 & 9.098 \\
HMWPE & 72.9 & 2.464 \\
PE & 122.4 & 4.344 \\
PP & 209.2 & 4.644 \\
NY66 & 801.9 & 4.145
\end{tabular}

at constant pressure. Cho et al. [17] also predicted a maximum in the thermal pressure coefficient with respect to temperature in the semi-crystalline polymer. These results obtained by Hartmann and Haque and Cho et al. [17] are consistent with the experimental data of the $\gamma_{V}$ vs. $V$ curve with a maximum at constant pressure obtained in this work.

\section{Conclusions}

In this work an empirical equation for the $\gamma_{V}$ vs. $V$ curve with a maximum at constant pressure has been determined based on the $P V T$ data available and empirical equations Eq. (1) for $V(P)$ and Eq. (2) for $V(T)$ in this work. An equation of state for zero internal pressure has been given by $P^{*} V^{*} / \mathrm{R} T^{*}=C^{*}-D^{*} V^{*}$ where the characteristic pressure $P^{*}$, volume $V^{*}$ and temperature $T^{*}$ are the values at which $\gamma_{V} T-P=0$, respectively, and $C^{*}$ and $D^{*}$ are constant.

\section{References}

[1] Pastine DJ. J Chem Phys 1968;49:3012

[2] Pastine DJ. J Appl Phys 1970;41:5085.

[3] Simha R, Jain RK. J Polym Sci Polym Phys Ed 1978;16:1471.

[4] Midha YR, Nanda VS. Macromolecules 1977;10:1031.

[5] Olabisi O, Simha R. Macromolecules 1975;8:206.

[6] Zoller P. J Appl Polym Sci 1977;21:3129.

[7] Anderson MS, Swenson CA. J Phys Chem Solids 1975;36(145).

[8] Syassen K, Holzapfel WB. Phys Rev B 1978;18:5826.

[9] Bernardes N, Swenson CA. In: Warschauer D, Paul W, editors. Solids under pressure. New York: McGraw-Hill; 1963.

[10] Saeki S, Tsubokawa M, Yamaguchi T. Polymer 1988;29:123.

[11] Saeki S, Tsubokawa M, Yamaguchi T. Polymer 1989;30:673.

[12] Saeki S, Tsubokawa M, Yamanaka J, Yamaguchi T. Polymer 1991; $32 ; 3170$.

[13] Wang F. Doctoral Dissertation, Department of Materials Science and Engineering, Faculty of Engineering, Fukui University; 1998.

[14] Saeki S. J Supercrit Fluids 1995;8:30.

[15] Wang F, Saeki S, Yamaguchi T. Polymer 1998;39:2235.

[16] Hartmann B, Haque MA. J Appl Phys 1985;58:2831.

[17] Seo MP, Shin BY, Ham DH, Cho B. J Appl Polym Sci 1995;58:143.

[18] He J, Zoller P. J Polym Sci Part B Polym Phys 1994;32:1049.

[19] Starkweather Jr HW, Jones GA, Zoller P. J Polym Sci Part B Polym Phys 1988;26:256

[20] Sears FW, Salinger GL. Thermodynamics, kinetic theory and statistical mechanics. Addison-Wesley Publishing Co.; 1975. 